\title{
Delay of early postnatal development as a risk factor for accelerated aging and Alzheimer's disease
}

\author{
Ekaterina Rudnitskaya \\ Molecular mechanisms of aging \\ Institute of Cytology and Genetics \\ SB RAS \\ Novosibirsk, Russia \\ rudnickaya@bionet.nsc.ru \\ Natalia Stefanova \\ Molecular mechanisms of aging \\ Institute of Cytology and Genetics \\ SB RAS \\ Novosibirsk, Russia \\ stefanovan@bionet.nsc.ru
}

\author{
Tatiana Kozlova \\ Molecular mechanisms of aging \\ Institute of Cytology and Genetics \\ SB RAS \\ Novosibirsk, Russia \\ kozlova@bionet.nsc.ru \\ Nataliya Kolosova \\ Molecular mechanisms of aging \\ Institute of Cytology and Genetics \\ SB RAS \\ Novosibirsk, Russia \\ kolosova@bionet.nsc.ru
}

\author{
Alena Burnyasheva \\ Molecular mechanisms of aging \\ Institute of Cytology and Genetics \\ SB RAS \\ Novosibirsk, Russia \\ burnyasheva@bionet.nsc.ru
}

\begin{abstract}
Alzheimer's disease (AD) is the most common cause of dementia worldwide. In the case of sporadic form of AD ( $95 \%$ of cases), its mechanisms are unknown. Accumulating data indicate middle age as a critical period for the relevant pathological processes; however, it has been reported only recently that in the early postnatal period - when brain development is completing-preconditions for a decrease in cognitive abilities and for accelerated aging can form. Here, we hypothesized that specific features of early postnatal brain development may be considered some of the prerequisites of $A D$ development at an advanced age. To test this hypothesis, we used OXYS rats, which are a suitable model of sporadic $A D$. The duration of gestation was lower in OXYS rats compared to control Wistar rats which may result in developmental retardation. Indeed, we noted retardation of emergence of postural and locomotor skills as well as decreased locomotor activity and increased anxiety in OXYS rats already at a young age: possible signs of altered brain development. We demonstrated retardation of the peak of postnatal neurogenesis in the hippocampal dentate gyrus (DG) of OXYS rats. Delayed neuronal maturation led to alterations of mossy-fiber formation and was accompanied by altered astrocytic migration from DG. We assume that the observed retardation in the development of the brain may be predictor of AD signs development in OXYS rats and, possibly, these diseases in humans.
\end{abstract}

Keywords - neurogenesis, brain development, Alzheimer's disease, OXYS rats

\section{Introduction}

Aging is the major risk factor for development of wide range of psychiatric disorders including AD. The most common $(\sim 95 \%$ of cases $)$ sporadic AD develops asymptomatically for many years prior to its manifestation [1] and accumulating data indicate middle age as a critical period for the relevant pathological processes [2, 3]; however, the question of when $\mathrm{AD}$ starts to develop remains open. It has been reported only recently that in the early postnatal periodwhen brain development is completing - preconditions for a decrease in cognitive abilities and for accelerated aging can form [4-6]. Evidently, this hypothesis cannot be verified without using adequate animal models. Previously we have confirmed that senescence-accelerated OXYS rats are a suitable model of sporadic AD [7-10]. Thus, we suggested that the study of the specific features of early postnatal development using OXYS rats may shed a light on long-

This work was supported by the Russian Science Foundation (\# 19-1500044). lasting effects of alterations of brain development and its connection to manifestation of $\mathrm{AD}$ late in life.

\section{Materials and methods}

For evaluation the duration of gestation we used 3-monthsold virgin female OXYS and control Wistar rats and agematched males ( $\mathrm{n}=25$ per strain and gender). For evaluation of body and brain weight we used offspring from female OXYS and Wistar rats $(\mathrm{n}=4$ female rats for one time point: day of birth, postnatal day 1 (PND1), PND3, PND5, PND7, PND10, PND14, PND20, PND45). For analysis of physical development (auricle detachment, emergence of pelage and incisors, eye opening, dropping of the testes in male and vaginal opening in female rats) and emergence of postural and locomotor skills (elevation of head and shoulders, grasp reflex for fore and hind limbs, righting on surface and in midair, negative geotaxis, placing, jumping and jumping across cliff) we used offspring from 4 female OXYS and Wistar rats per 23 parameters. To evaluate locomotor activity we used open field test for animals at PND10, PND14, PND20 and PND45; to evaluate anxiety level we used elevated plus maze test for animals at PND45 ( $\mathrm{n}=15$ per strain and age). To analyze hippocampal development of OXYS and Wistar rats we evaluated the density of neuronal progenitors and proliferating cells in the DG at the day of birth, PND1, PND3, PND5, PND7, PND10, PND14, PND20 and PND45 as well as the density of neuronal and astroglial cell lines at PND10, PND14, PND20 and PND45 by immunochistochemistry; intensity of apoptosis at PND10, PND14, PND20 and PND45 by TUNEL method ( $\mathrm{n}=3-6$ per strain and age). To evaluate morphological characteristics of mossy fibers, hippocampal slices of OXYS and Wistar rats at PND10, PND14, PND21 and PND45 underwent DiI injection into the DG area $(n=6-$ 8 per strain and age) with following microscopic analysis. To clarify the connection between features of early development and AD-like pathology we evaluated the density of progenitors and cells from neuronal and astroglial cell lines in the DG of OXYS rats at period of active manifestation (age 3 months) and progression (age 18 months) of the disease signs. The data were subjected to two-way analysis of variance in the Statistica 8.0 software.

\section{Results}

We showed that duration of gestation was shorter in OXYS rats compared to Wistar rats, and OXYS pups were born with lower body as well as brain weight. At PND1 the 
body weight did not differ between OXYS and Wistar pups, whereas brain weight remained lower in OXYS pups. However, to PND3 the differences in brain weight between rat strains become insignificant. Shortened duration of gestation in OXYS rats may result in developmental retardation, such as delayed auricle detachment, emergence of pelage and incisors, eye opening, dropping of the testes in male rats and vaginal opening in female rats. Moreover, we observed retardation of emergence of postural and locomotor skills in OXYS rats: elevation of head and shoulders, grasp reflex for hind limbs, righting on surface and in midair, negative geotaxis, placing (when chin elicited) and jumping across cliff. Also we demonstrated decreased locomotor activity in OXYS rats at PND20 and PND45 and increased anxiety at PND45. These changes may reflect deterioration of brain development. Indeed, we demonstrated that OXYS pups were born with decreased density of amplifying neuronal progenitors in DG; however, to PND1 the parameter increased reaching the level of Wistar pups. The density of amplifying neuronal progenitors naturally decreased to PND10 in both rat strains; however, in OXYS pups the decrease was more pronounced: at PND10 the parameter was lower compared to Wistar pups; to PND14 the difference disappeared. The density of immature neurons was higher in DG of OXYS rats at PND10 which may indicate delay of postnatal peak of neurogenesis. Moreover, the peak of postnatal apoptosis was observed at PND20 in OXYS rats, which indicate its retardation. As for neurite development, the following features of mossy-fiber formation in OXYS rats were revealed by our colleagues [11]: a smaller suprapyramidal bundle and lager infrapyramidal bundle, less pronounced fasciculation of granule cell axons, and smaller size and an irregular shape of nuclei in the CA3 pyramidal layer. However, to PND45 these features of hippocampal morphology in OXYS rats disappeared, and the parameters became the same as in Wistar rats. Besides we found altered astrocytic migration from DG in OXYS rats. It's important to emphasize that there were long-lasting consequences of these abnormalities of postnatal hippocampal development in OXYS rats such as insufficiency of astrocytic support of hippocampal neurogenic niche at 3 months of age and lower intensity of neurogenesis during lifespan: indeed, the density of stem quiescence neuronal progenitors was higher in DG of OXYS rats at 18 months of age.

\section{Conclusion}

To conclude, in present work we observed shortened duration of gestation and, as a consequence, delayed postnatal development of OXYS rats. Behavioral abnormalities may reflect altered brain development. Indeed, peaks of postnatal neurogenesis and apoptosis in DG of OXYS rats fell later compared to Wistar rats. Moreover, formation of hippocampal mossy fibers was altered in OXYS rats. We suppose that the observed features of early hippocampal development have long-lasting effects on hippocampal plasticity and, thus, are the one of predictors of AD-like pathology in OXYS rats and may be one of risk factors for development of sporadic AD in human.

\section{ACKNOWLEDGMENT}

Senescence-accelerated OXYS rats and Wistar rats were obtained from the Breeding Experimental Animal Laboratory of the Institute of Cytology and Genetics, SB RAS,
Novosibirsk, Russia. The microscopy was conducted at the Multi-Access Center for Microscopy of Biological Objects (Institute of Cytology and Genetics, SB RAS, Novosibirsk, Russia).

\section{REFERENCES}

[1] M. Hersi, B. Irvine, P. Gupta, J. Gomes, N. Birkett, D. Krewski, "Risk factors associated with the onset and progression of Alzheimer's disease: A systematic review of the evidence," Neurotoxicology, vol. 61, 2017, pp. 143-187.

[2] R. J. Bateman, C. Xiong, T. L. Benzinger, A. M. Fagan, A. Goate, N. C. Fox, et al., "Clinical and biomarker changes in dominantly inherited Alzheimer's disease,” New England Journal Medicine, vol. 367, 2012, pp. 795-804.

[3] M. Crous-Bou, C. Minguillón, N. Gramunt, J. L. Molinuevo, "Alzheimer's disease prevention: from risk factors to early intervention," Alzheimers Research Therapy, vol. 9, 2017, No. 71.

[4] K. Heinonen, J. G. Eriksson, J. Lahti, E. Kajantie, A. K. Pesonen, S. Tuovinen, et al., "Late preterm birth and neurocognitive performance in late adulthood: a birth cohort study," Pediatrics, vol. 135, 2015, pp. $818-825$.

[5] N. N. Nalivaeva, A. J. Turner, I. A. Zhuravin, "Role of Prenatal Hypoxia in Brain Development, Cognitive Functions, and Neurodegeneration," Frontiers Neuroscience, vol. 12, 2015, No. 825.

[6] L. K. Axelrud, J. R. Sato, M. L. Santoro, F. Talarico, D. S. Pine, L. A. Rohde, et al., "Genetic risk for Alzheimer's disease and functional brain connectivity in children and adolescents," Neurobiology Aging, vol. 82, 2019, pp. 10-17.

[7] N. A. Stefanova, O. S. Kozhevnikova, A. O. Vitovtov, K. Y. Maksimova, S. V. Logvinov, E. A. Rudnitskaya, E. E. Korbolina, N. A. Muraleva, N. G. Kolosova, "Senescence-accelerated OXYS rats: A model of age-related cognitive decline with relevance to abnormalities in Alzheimer disease," Cell Cycle, vol. 13, 2014, pp. 898-909.

[8] N. A. Stefanova, K. Y. Maksimova, E. A. Rudnitskaya, N. A. Muraleva, N. G. Kolosova, "Association of cerebrovascular dysfunction with the development of Alzheimer's disease-like pathology in OXYS rats," BMC Genomics, vol. 19, 2018, No. 75.

[9] N. A. Stefanova, N. I. Ershov, K. Y. Maksimova, N. A. Muraleva, M. A. Tyumentsev, N. G. Kolosova, "The Rat Prefrontal-Cortex Transcriptome: Effects of Aging and Sporadic Alzheimer's DiseaseLike Pathology," Journals Gerontology: Series A Biological Sciences Medical Sciences, vol. 74, 2019, pp. 33-43.

[10] E. A. Rudnitskaya, T. A. Kozlova, A. O. Burnyasheva, N. G. Kolosova, N. A. Stefanova, "Alterations of hippocampal neurogenesis during development of Alzheimer's disease-like pathology in OXYS rats," Experimental Gerontology, vol. 115, 2019, pp. 32-45.

[11] E. A. Rudnitskaya, T. A. Kozlova, A. O. Burnyasheva, A. E. Tarasova, T.M. Pankova, M. V. Starostina, N. A. Stefanova, N. G. Kolosova, "Alteration of postnatal hippocampal development as a potential risk factor for Alzheimer's disease-like pathology in OXYS rats," Frontiers Neuroscience, 2020, in press. 